\title{
TITLE:
}

\section{Analog frequency modulation detector for dynamic force microscopy}

\section{$\operatorname{AUTHOR}(\mathrm{S})$ :}

Kobayashi, Kei; Yamada, Hirofumi; Itoh, Hiroshi; Horiuchi, Toshihisa; Matsushige, Kazumi

\section{CITATION:}

Kobayashi, Kei ...[et al]. Analog frequency modulation detector for dynamic force microscopy. REVIEW OF SCIENTIFIC INSTRUMENTS 2001, 72(12): 4383-4387

\section{ISSUE DATE:}

2001-12

URL:

http://hdl.handle.net/2433/39804

\section{RIGHT:}

Copyright 2001 American Institute of Physics. This article may be downloaded for personal use only. Any other use requires prior permission of the author and the American Institute of Physics. 


\title{
Analog frequency modulation detector for dynamic force microscopy
}

\author{
Kei Kobayashi, ${ }^{\text {a) }}$ Hirofumi Yamada, Hiroshi Itoh, ${ }^{\text {b) }}$ Toshihisa Horiuchi, and Kazumi \\ Matsushige \\ Department of Electronic Science and Engineering, Kyoto University, Kyoto 606-8501, Japan
}

(Received 7 March 2001; accepted for publication 17 September 2001)

\begin{abstract}
A new analog frequency modulation (FM) detector (demodulator) for dynamic force microscopy (DFM) is presented. The detector is designed for DFM by utilizing the FM detection method where the resonance frequency shift of the force sensor is kept constant to regulate the distance between a tip and a sample surface. The FM detector employs a phase-locked loop (PLL) circuit using a voltage-controlled crystal oscillator (VCXO) so that the thermal drift of the output signal is negligibly reduced. The PLL is used together with a frequency conversion (heterodyne) circuit allowing the FM detector to be used for a wide variety of force sensors with the resonance frequency ranging from $10 \mathrm{kHz}$ to $10 \mathrm{MHz}$. The minimum detectable frequency shift was as small as $0.1 \mathrm{~Hz}$ at the detection bandwidth of $1 \mathrm{kHz}$. The detector can track a resonance frequency shift as large as $1 \mathrm{kHz}$. We also present some experimental results including the observations of the $\mathrm{Si}(111)-7 \times 7$ reconstructed surface and fullerene molecules deposited on the surface by DFM using this FM detector. () 2001 American Institute of Physics. [DOI: 10.1063/1.1416104]
\end{abstract}

\section{INTRODUCTION}

\section{A. Dynamic force microscopy (DFM)}

Since a vibrating force sensor in dynamic force microscopy (DFM) (Refs. 1-3) is scanned over the surface without a static contact, the technique is a powerful tool especially for soft samples for which the tip perturbation has to be reduced in order to avoid damaging surface structures. In DFM, a force sensor such as a cantilever with a tip is vibrated at or near the resonance frequency. When the tip is brought close enough to the sample, the resonance frequency is shifted due to a tip-sample interaction. The resulting resonance frequency shift can be used to regulate the distance between the tip and the sample.

There are two major methods to detect such a shift in the resonance frequency of the force sensor in DFM. The most common method in an ambient condition is the amplitude detection method, ${ }^{2}$ where the force sensor is vibrated at a frequency near the resonance frequency and the change in the vibration amplitude due to the resonance frequency shift is kept constant to regulate the tip-sample distance. Though an experimental setup for the amplitude detection method is very simple, the method suffers from a long relaxation time when a force sensor with a high $Q$ factor is used. In particular, the long relaxation time often becomes a serious problem in an environment such as a vacuum environment, where the $Q$ factor typically reaches 10000 . The frequency modulation (FM) detection method ${ }^{3}$ is a solution to this problem. In the FM detection method, the force sensor is kept oscillated at its resonance frequency by positive feedback loop electronics. The shift of the resonance frequency is monitored by using

\footnotetext{
a) Present address: International Innovation Center, Kyoto University, Kyoto 606-8501, Japan; electronic mail: keicoba@iic.kyoto-u.ac.jp

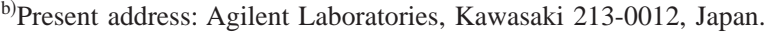

the FM detector and is kept constant to regulate the gap distance.

The FM detection method has been rapidly common for DFM in an ultra-high-vacuum (UHV) condition. In this case, a slight negative frequency shift of the vibrating cantilever is gently controlled and used to obtain highly resolved images. Following the first success of taking an atomically resolved image of the $\mathrm{Si}(111)-7 \times 7$ reconstructed surface, ${ }^{4}$ a large number of clear images on semiconductors, ${ }^{5,6}$ alkali halides, ${ }^{7}$ and molecules ${ }^{8,9}$ have been obtained by DFM utilizing the FM detection method.

\section{B. Frequency modulation (FM) detector}

The FM detector is one of the critical instruments in the experimental setup for the FM detection method. Among various detectors used for the FM detection method, the most common detectors are a quadrature detector and a phaselocked loop (PLL) detector. ${ }^{10-12}$ Considering the practical applications in DFM, the working frequency range is a very important factor to design the FM detector.

In the case of the quadrature detector, the working frequency range is very narrow so that the circuit parameters must be adjusted for a different force sensor with a varying resonance frequency is changed unless a frequency conversion (heterodyne) circuit is utilized. On the other hand, in the case of the PLL detector, the working frequency range is determined by a tuning range of a voltage-controlled oscillator (VCO) in the PLL. Thus it is very easy to implement the PLL detector that can be used for a wide variety of force sensors with any resonance frequency without changing circuit parameters by utilizing VCO with a wide tuning range. For this reason, the PLL detector is often equipped for the FM detection method in commercially available DFM instruments. In addition, PLL detectors have some advantages be- 


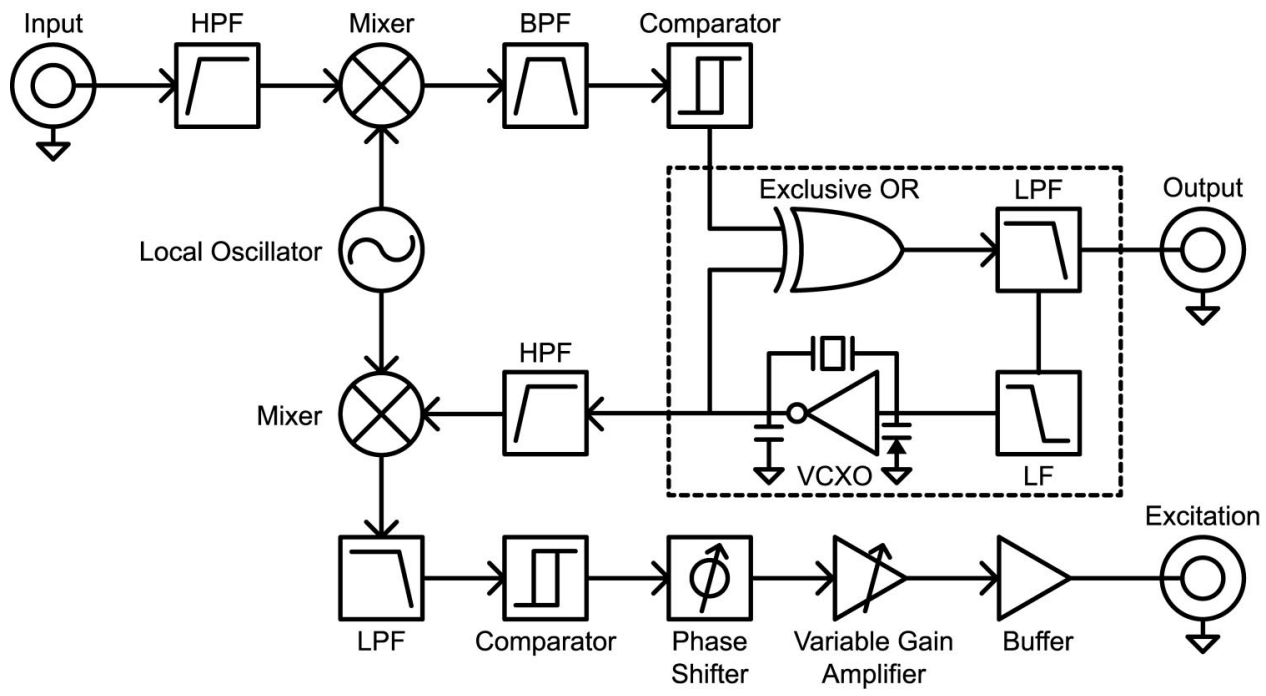

FIG. 1. Schematic of an analog frequency modulation detector. The detector consists of a frequency conversion circuit and a phase-locked loop (PLL) using a voltage-controlled crystal oscillator (VCXO). The PLL block is indicated by a dashed rectangle. The frequency of an input signal $\left(f_{0}\right)$ is converted to the intermediate frequency (IF), which is $4.5 \mathrm{MHz} \pm 450$ $\mathrm{Hz}$, by frequency mixing with the output signal of the local oscillator. The output signal of the VCXO can be used to make an excitation signal by another frequency mixing.

cause the output signal of the VCO can be used to excite the force sensor. $^{13}$

Since the dominant noise source in each detector is the phase noise of the phase detector, the sensitivity is the same for each detector in principle. Although many integrated circuits (ICs) designed to implement the PLL detector are commercially available, their specifications do not satisfy DFM applications since integrated VCOs in these ICs often have a large thermal drift in the output frequency. Such a large thermal drift is due to the fact that the output frequency of the $\mathrm{VCO}$ is determined by passive electrical components.

We consider that the PLL detector utilizing a highly stable VCO is one of the ideal FM detector. We also consider that the VCO can be thermally stabilized using a frequencystabilizing element such as a crystal resonator or a ceramic resonator with a high $Q$ factor. It should be noted that another solution is to use digital circuits to implement the VCO,${ }^{14,15}$ which is also stabilized using a crystal oscillator generating a clock signal.

In this article we introduce a new FM detector using a voltage-controlled crystal oscillator (VCXO) as the VCO in the PLL in order to decrease the thermal drift. In this detector, the PLL is used together with a frequency conversion circuit allowing the FM detector to be used for a wide variety of force sensors. An implementation of the FM detector and some experimental results obtained using the detector are described.

\section{CIRCUIT DESIGN}

Figure 1 shows a block diagram of a newly developed FM detector. The frequency of the input signal, which comes from a displacement sensor is the resonance frequency of the force sensor $\left(f_{0}\right)$ in a self-oscillation loop. The PLL block is indicated by a dashed rectangle in Fig. 1. The PLL block consists of three components, which are a phase detector (PD), a loop filter (LF), and the VCXO, which is used as a thermally stable VCO.

Since the working frequency range of the PLL is limited by a very narrow tuning range of $\mathrm{VCXO}$, the frequency of the input signal to the PLL $\left(f_{0}\right)$ should be converted to an intermediate frequency (IF), which is within the working frequency range of the PLL. When a small shift in the input frequency is caused, the IF also shifts by the same amount and then the shift is measured as the control voltage of the VCXO in the PLL, which is fed to the feedback electronics to regulate the gap distance. Moreover, the output frequency of the VCXO can be used for an excitation of the cantilever vibration after another frequency conversion to the input frequency.

In DFM, the tip-sample distance is regulated by maintaining a small frequency shift constant. The shift can be negative or positive depending on the tip position (attractive regime or repulsive regime, respectively). Since the amount of the frequency shift ranges from several hertz to several hundred hertz for typical DFM operating conditions, we decided to use an operating frequency range of the PLL of about $1 \mathrm{kHz}$. The typical tuning range for the VCXO is several hundred ppm of the center frequency, and thus we decided to use the VCXO with the center frequency of several megahertz. An effective bandpass filter is required in the frequency conversion circuit to eliminate the undesirable frequency spectrum other than the signal component around the IF. A frequency of $4.5 \mathrm{MHz}$ was chosen as the IF since a ceramic bandpass filter with the center frequency of $4.5 \mathrm{MHz}$ was commercially available. ${ }^{16}$

\section{A. Phase-locked loop (PLL) circuit}

A high-speed exclusive OR logic gate IC (Texas Instruments: SN74F86), ${ }^{17}$ which is followed by an active low-pass (Sallen-and-Key 2-pole) filter with a unity gain, is used as the PD. The active low-pass filter (LPF) is implemented by using an operational amplifier (Burr-Brown: OPA227). ${ }^{17}$ The cutoff frequency of the LPF is set at about $100 \mathrm{kHz}$.

An active filter implemented by using OPA227 is used for the LF. When the maximum modulation frequency of the VCXO is sufficiently high, the detection bandwidth of the PLL can be raised by increasing the loop gain. However, it should be noted that the phase noise from the PD appears as the amplitude noise in the output signal of the PLL thus a large loop gain consequently increases the amplitude noise in the demodulated signal. Since a lateral resolution of 
the DFM can be improved by eliminating long-range forces such as an electrostatic force (ESF), the DFM image is obtained while the ESF is canceled out by compensating a contact potential difference (CPD) between a tip and a sample surface. For the compensation of the CPD, an ac modulation voltage is applied and then an induced resonance frequency modulation due to ESF is detected by a lock-in technique. For this modulation technique called Kelvin probe force microscopy (KFM), ${ }^{18,19}$ the demodulation bandwidth of the FM detector should be sufficiently high.

For the VCO in the PLL, a commercial VCXO (Champion Technologies: K1525), ${ }^{20}$ with the center frequency of 4.5 $\mathrm{MHz}$, is used. The nominal frequency deviation is as large as $\pm 100 \mathrm{ppm}( \pm 450 \mathrm{~Hz})$ with a control voltage ranging from 0.5 to $4.5 \mathrm{~V}$ (a voltage span of $4.0 \mathrm{~V}$ ). This means that the frequency sensitivity of the PLL using this VCXO is about $4.4 \mathrm{mV} / \mathrm{Hz}$. The nominal modulation bandwidth of the VCXO is more than $20 \mathrm{kHz}$, which is high enough to implement the PLL with a realistic demodulation bandwidth for KFM. Since the output signal of the VCXO is a square wave $(0.5-4.5 \mathrm{~V})$, the signal is directly fed to an input of the PD.

Finally, another implementation of the PLL block can be realized with a commercial PLL IC (Motorola: MC44144), ${ }^{21}$ which was originally designed for application in a video system. The IC is composed of the PD, a user-configurable laglead passive filter as the LF, and the VCXO. The center frequency of the PLL can be configured to $4.5 \mathrm{MHz}$ by using an external crystal resonator of $18 \mathrm{MHz}$, which is four times as large as the center frequency.

\section{B. Frequency conversion circuit}

The frequency of an input signal $\left(f_{0}\right)$ is converted to the IF $\left(f_{0}^{\prime}\right)$ by mixing with the frequency of the signal from an local oscillator $\left(f_{l}\right)$. The input signal coming from a displacement sensor is fed to an input $(X)$ of an analog multiplier (Analog Devices: AD734), ${ }^{22}$ whose function is expressed as $W=X Y / U$ where $X, Y$, and $U$ denote three input voltages and $W$ is the output voltage. Since the frequency stability of the local oscillator needs to be better than that of the VCXO, it should be stable so that a frequency synthesizer (Digital Signal Technology: PCK-50), ${ }^{23}$ based on direct digital synthesis (DDS), is used. The signal from the local oscillator whose amplitude is $1.0 \mathrm{~V}$ peak to peak is fed to another input $(Y)$ of the multiplier.

The frequency of an output signal from the local oscillator $\left(f_{l}\right)$ should be chosen in a way that the $\operatorname{IF}\left(f_{0}^{\prime}\right)$ is located in the working frequency range of the PLL, which is 4.5 MHz $\pm 450 \mathrm{~Hz}$. Depending on both the specifications of the feedback electronics and the polarity of the frequency shift (positive or negative) to regulate the gap distance, $f_{l}$ can be chosen as $f_{0}^{\prime}+f_{0}$ or $\left|f_{0}^{\prime}-f_{0}\right|$, thus $f_{l}$ should be in the range $\left(4.5 \mathrm{MHz}+f_{0}\right) \pm 450 \mathrm{~Hz}$ or $\left|4.5 \mathrm{MHz}-f_{0}\right| \pm 450 \mathrm{~Hz}$.

The output signal of the multiplier includes two different frequency components. One is at the IF $\left(f_{0}^{\prime}\right)$ and the other frequency component exists at $f_{0}^{\prime}+2 f_{0}$ or $\left|f_{0}^{\prime}-2 f_{0}\right|$ for the case using $f_{0}^{\prime}+f_{0}$ or $\left|f_{0}^{\prime}-f_{0}\right|$ as $f_{l}$, respectively. Only the frequency component at the IF is selected by using an efficient ceramic bandpass filter (Murata Manufacturing:
SFE4.5MB) ${ }^{16}$ If a frequency component other than the IF $\left(f_{0}^{\prime}\right)$ is not suppressed with this bandpass filter, the PD produces the beat signal by mixing it with the output signal of the VCXO. The frequency of the beat signal is $2 f_{0}$ or $2 f_{0}^{\prime}$ $+2 f_{0}$ in the case using $f_{0}^{\prime}+f_{0}$ as $f_{l}$ and $2 f_{0}$ or $\left|2 f_{0}^{\prime}-2 f_{0}\right|$ in the case using $\left|f_{0}^{\prime}-f_{0}\right|$ as $f_{l}$, respectively. This may cause a problem because the frequency of the beat signal is comparable with the detection bandwidth of the PLL when $f_{0}$ is lower than $10 \mathrm{kHz}$ or located within $f_{0}^{\prime}(4.5 \mathrm{MHz}) \pm 10 \mathrm{kHz}$. Otherwise, it does not cause any problem even without the efficient bandpass filter.

Then, the output signal of the bandpass filter is fed into a comparator (Analog Devices: AD790), ${ }^{22}$ which converts a sinusoidal wave to a square wave $(0.5-4.5 \mathrm{~V})$ to meet the requirement of the PLL, where the high-speed exclusive OR logic gate IC is used as the PD.

\section{Excitation output}

In the experimental setup for DFM utilizing the FM detection method, positive feedback electronics is required in order to keep the cantilever vibrating at the resonance frequency, The most common method to implement positive feedback electronics is to utilize the displacement signal directly as an excitation signal through a phase shifter and a variable gain amplifier. Another method is to use the VCO output signal, which is phase locked to the input frequency. ${ }^{13}$ In the latter method, the free-running frequency of the VCO can be very close to the cantilever resonance frequency if the tuning range of the VCO is sufficiently narrow. In other words, the cantilever vibration can be still excited near its resonance frequency even when the cantilever vibration motion is accidentally suppressed. Furthermore, this is also a great advantage for operation in an environment where the $Q$ factor of the cantilever is very low. The cantilever with a low $Q$ factor in the self-oscillation loop often starts to vibrate at an undesired frequency, which is not the same as the resonance frequency. However, by using the VCO output as a source of the excitation signal, the excitation signal is always located near $f_{0}( \pm 450 \mathrm{~Hz}$ for our detector), even during the time when no input signal is fed to the FM detector. In addition, by using an automatic gain control (AGC) circuit, the stability of the positive feedback electronics could even be improved.

For another frequency conversion of the IF $\left(f_{0}^{\prime}\right)$ back to $f_{0}$, the output signal of the VCXO is fed into a multiplier (AD734) through a high-pass filter (HPF). The signal from the local oscillator is also fed into the multiplier.

In order to control the phase of the excitation signal, we used a phase shifter module (NF: CD-951V4), ${ }^{24}$ which can shift the phase of the excitation signal by a suitable amount within $\pm 180^{\circ}$. Since the maximum input frequency of the phase shifter is $2 \mathrm{MHz}$, the upper limit of the frequency of the excitation signal is limited by the phase shifter.

In between the multiplier and the phase shifter, there is an active LPF and a comparator. The LPF is used to suppress the higher-frequency component other than the excitation frequency component and the comparator is used to convert 


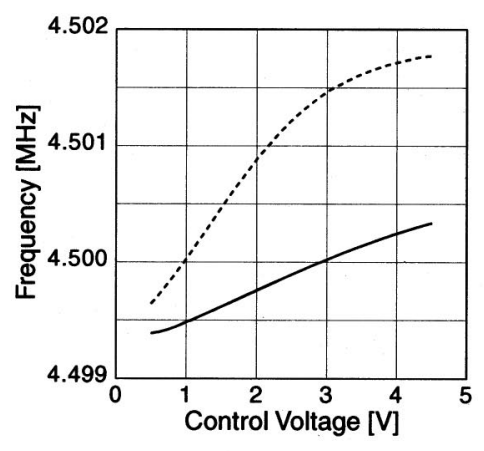

(a)

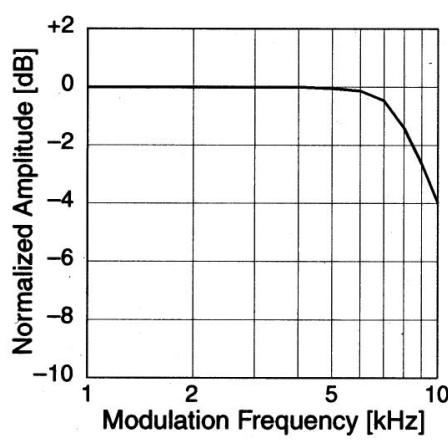

(b)

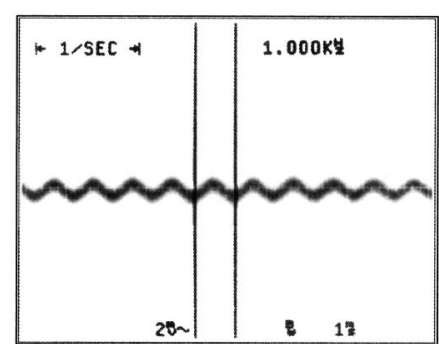

Modulation Frequency: $1 \mathrm{kHz}$ Frequency Deviation: $0.1 \mathrm{~Hz}$ peak-to-peak

(c)

FIG. 2. (a) Plot of the output frequency of the VCXO as a function of the control voltage. Solid curve shows the output frequency of the VCXO (K1525). The curve shows the linear characteristics for a frequency span of about $900 \mathrm{~Hz}$. Dashed curve is a measured characteristic of the VCXO in the PLL IC (MC44144) with the external crystal resonator (18 MHz). (b) Plot of the normalized amplitude of the demodulated signal as a function of the modulation frequency of the input signal. The modulation frequency ranged from 1 to $10 \mathrm{kHz}$ and the frequency deviation was set to $1.0 \mathrm{~Hz}$ peak to peak. (c) Oscilloscope photograph of the output signal of the detector when a frequency-modulated sine wave with a maximum frequency deviation of $0.1 \mathrm{~Hz}$ peak to peak was used as the input signal to the FM detector. The modulation frequency was fixed to $1.0 \mathrm{kHz}$.

the wave form of the excitation signal for the following phase shifter.

Then, amplitude of the excitation signal is controlled by a voltage-controlled-gain amplifier implemented by using a multiplier (AD734). The output signal of the phase shifter is fed into an input $(X)$ of the multiplier while a dc control voltage is fed to another input $(Y)$. Since the output function is $W=X Y / U$, the excitation amplitude can be controlled by a dc control voltage $(Y)$ while the divider input $U$ is fixed (excitation amplitude constant mode). Furthermore, if an appropriate dc control voltage generated by another feedback electronics is supplied to the divider input $U$ to regulate the vibration amplitude, one can measure the dissipation of the cantilever vibration energy occurring from the complex tipsample interaction forces (constant vibration amplitude mode). Finally, a high-speed unity-gain buffer (Burr-Brown: OPA633) (Ref. 17) is used to supply an excitation signal.

\section{EXPERIMENTAL RESULTS}

\section{A. FM detector performance}

For studying the performance of the FM detector, the fundamental characteristics of the PLL were measured and DFM imaging in a vacuum environment was carried out. Figure 2(a) shows a measured input-output characteristic of the VCXO in the PLL. The solid curve shows the output frequency of the VCXO (K1525) plotted as a function of the control voltage ranging from 0.5 to $4.5 \mathrm{~V}$. The curve shows a good linearity for a frequency span of about $900 \mathrm{~Hz}$. The characteristic of the VCXO in the PLL IC (MC44144) with the external crystal resonator $(18 \mathrm{MHz})$ is also shown as a dashed curve for comparison.

Then, the demodulation bandwidth of the FM detector was measured. We used an arbitrary function generator (Tektronix: AFG310) (Ref. 25) for supplying the test signal. The test signal was a frequency-modulated sinusoidal wave with the carrier frequency of $300 \mathrm{kHz}$ with a maximum deviation of $1.0 \mathrm{~Hz}$ peak to peak. The carried signal was a sinusoidal wave, whose frequency ranged from 1 to $10 \mathrm{kHz}$. The frequency of the local oscillator $\left(f_{l}\right)$ was set to $4.2 \mathrm{MHz}$. Figure 2(b) shows a measured curve of the amplitude of the sinusoidal output signal detected by the PLL block as a function of the modulation frequency of the input signal. The amplitude was plotted in the vertical axis normalized for the lowfrequency response, which means that $0 \mathrm{~dB}$ corresponds to about $4.4 \mathrm{mV} / \mathrm{Hz}$. As we can see in Fig. 2(b), the bandwidth $(-3 \mathrm{~dB})$ is about $10 \mathrm{kHz}$.

Figure 2(c) is an output signal trace of the FM detector observed with an oscilloscope (Tektronix: 2245A). ${ }^{26}$ The maximum deviation was reduced to $0.1 \mathrm{~Hz}$ peak to peak in this case and the modulation frequency was fixed to $1 \mathrm{kHz}$. We can see that the circuit stably follows a frequency deviation of $0.1 \mathrm{~Hz}$ peak to peak at the detection bandwidth of 1 $\mathrm{kHz}$. This fact means that we can detect a force derivative of $0.02 \mathrm{mN} / \mathrm{m}$ at the bandwidth of $1 \mathrm{kHz}$ when we use a cantilever with a spring constant $(k)$ of $30 \mathrm{~N} / \mathrm{m}$ and a resonance frequency of $300 \mathrm{kHz}$ and when we assume that the frequency shift corresponds to the change in the force gradient expressed as follows: $\Delta f_{0} / f_{0}=(\partial F / \partial z) / 2 k$.

\section{B. DFM application}

The FM detector was used with a commercial DFM instrument (JEOL: JAFM-4500XT) ${ }^{26}$ The displacement of a vibrating cantilever was detected with a typical beam deflection method. The cantilever used was a commercial Si cantilever (Nanosensors: $\mathrm{NCH}$ ). ${ }^{27}$ The typical spring constant and the resonance frequency were $30 \mathrm{~N} / \mathrm{m}$ and $300 \mathrm{kHz}$, respectively. The measured $Q$ factor of the cantilever was about 30000 . The estimated vibration amplitude was about $10 \mathrm{~nm}$ peak to peak.

The $\operatorname{Si}(111)-7 \times 7$ reconstructed surface was prepared by flashing a Si strip at $1200^{\circ} \mathrm{C}$. Figure 3(a) shows a DFM image of the $\mathrm{Si}(111)-7 \times 7$ reconstructed surface using the FM detector described here. The frequency shift was kept constant at $-20 \mathrm{~Hz}$ during imaging. Furthermore, fullerene $\left(\mathrm{C}_{60}\right)$ molecules were deposited onto the $\mathrm{Si}(111)-7 \times 7$ surface from a crucible heated at $370{ }^{\circ} \mathrm{C}$. A DFM image of the fullerene molecules, as shown in Fig. 3(b), was obtained while the frequency shift was kept constant at $-50 \mathrm{~Hz}$. The image was taken on the terrace of a fullerene crystalline is- 


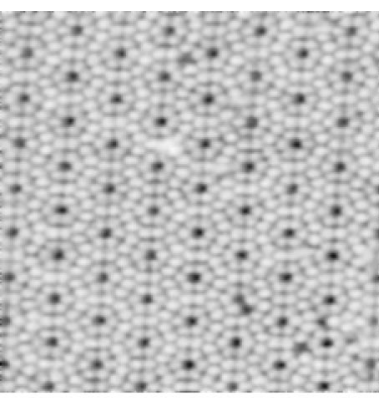

(a)

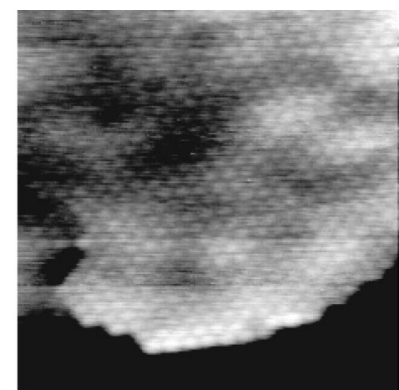

(b)
FIG. 3. Dynamic force microscopy images obtained using the FM detector. (a) $20 \mathrm{~nm} \times 20 \mathrm{~nm}$ image of the $\mathrm{Si}(111)-7 \times 7$ reconstructed surface $(\Delta f=$ $-20 \mathrm{~Hz}$ ). (b) $25 \mathrm{~nm} \times 25 \mathrm{~nm}$ image of the $\mathrm{C}_{60}$ molecules deposited on the $\operatorname{Si}(111)-7 \times 7$ surface $(\Delta f=-50 \mathrm{~Hz})$.

land so that the dark region in Fig. 3(a) is lower than the terrace by $2.0 \mathrm{~nm}$ (two molecular layers). Thus, the high performance of the PLL detector was successfully demonstrated.

\section{ACKNOWLEDGMENTS}

This work was supported by a Grant-in-Aid for Scientific Research from the Ministry of Education, Science, Sports and Culture of Japan. The authors would like to acknowledge Kyoto University Venture Business Laboratory Project and Kansai Technology Licensing Organization Co., Ltd.

${ }^{1}$ Y. Martin, C. C. Williams, and H. K. Wickramasinghe, J. Appl. Phys. 61, 4723 (1987).

${ }^{2}$ Q. Zhong, D. Inniss, K. Kjoller, and V. B. Elings, Surf. Sci. Lett. 290, L688 (1993).
${ }^{3}$ T. R. Albrecht, P. Grütter, D. Horne, and D. Rugar, J. Appl. Phys. 69, 668 (1991)

${ }^{4}$ F. J. Giessibl, Science 267, 68 (1995).

${ }^{5}$ S. Kitamura and M. Iwatsuki, Jpn. J. Appl. Phys., Part 2 34, L145 (1995).

${ }^{6}$ Y. Sugawara, M. Ohta, H. Ueyama, and S. Morita, Science 270, 1646 (1995).

${ }^{7}$ M. Bammerlin, R. Lüthi, E. Meyer, A. Baratoff, J. Lü, M. Guggisberg, C. Gerber, L. Howald, and H.-J. Güntherodt, Probe Microsc. 1, 3 (1997).

${ }^{8}$ B. Gotsmann, C. Schmidt, C. Seidel, and H. Fuchs, Eur. Phys. J. B 4, 267 (1998).

${ }^{9}$ K. Kobayashi, H. Yamada, T. Horiuchi, and K. Matsushige, Appl. Surf. Sci. 140, 281 (1999).

${ }^{10}$ F. M. Gardner, Phaselock Technique, 2nd ed. (Wiley, New York, 1979).

${ }^{11}$ R. E. Best, Phase-Locked Loops, 4th ed. (McGraw-Hill, New York, 1999).

${ }^{12}$ G. Nash, Motorola Application Note No. AN535 (1994).

${ }^{13}$ U. Dürig, H. R. Steinauer, and N. Blanc, J. Appl. Phys. 82, 3641 (1997).

${ }^{14}$ H. Edwards, L. Taylor, W. Duncan, and A. J. Melmed, J. Appl. Phys. 82, 980 (1997).

${ }^{15}$ Ch. Loppacher, M. Bammerlin, F. Battiston, M. Guggisberg, D. Müller, H. R. Hidber, R. Lüthi, E. Meyer, and H.-J. Güntherodt, Appl. Phys. A: Mater. Sci. Process. 66, S215 (1998).

${ }^{16}$ Murata Manufacturing Co., Ltd., Kyoto, Japan (http://www.murata.com).

${ }^{17}$ Texas Instruments, Inc., Dallas, TX (http://www.ti.com).

${ }^{18}$ S. Kitamura and M. Iwatsuki, Appl. Phys. Lett. 72, 3154 (1998).

${ }^{19}$ K. Kobayashi, H. Yamada, T. Horiuchi, and K. Matsushige, Appl. Surf. Sci. 157, 281 (2000).

${ }^{20}$ Champion Technologies, Inc., Franklin Park, IL (http:// www.champtech.com).

${ }^{21}$ Motorola, Inc., Schaumburg, IL (http://www.mot.com).

${ }^{22}$ Analog Devices, Inc., Norwood, MA (http://www.analog.com).

${ }^{23}$ Digital Signal Technology, Inc., Asaka, Japan (http://www.dst.co.jp/ index_us.html).

${ }^{24} \mathrm{NF}$ Corporation, Yokohama, Japan (http://www.nfcorp.co.jp/english/ index.htm).

${ }^{25}$ Tektronix, Inc., Beaverton, OR (http://www.tek.com).

${ }^{26}$ JEOL Ltd., Akishima, Japan (http://www.jeol.com).

${ }^{27}$ NANOSENSORS GmbH, Wetzlar-Blankenfeld, Germany (http:// www.nanosensors.com). 